\title{
Metaphors for Supporting User Interaction with Multimedia Databases - A Demonstration
}

\author{
M. F. Costabile, D. Malerba
}

Dipartimento di Informatica, Università degli Studi di Bari

Via Orabona 4, I-70126 Bari, Italy

\{costabile,malerba\}@di.uniba.it

\author{
M. Hemmje, A. Paradiso \\ Integration Information Systems Institute, GMD \\ Dolivostrasse 4, D-64293 Darmstadt, Germany \\ \{hemmje,paradiso\}@darmstadt.gmd.de
}

\begin{abstract}
This demonstration presents a prototype system in which metaphors for supporting user interaction with multimedia databases are automatically generated. Such a prototype is a further step in the development of Virgilio, a Virtual Reality (VR) based system that has been designed to be a general purpose exploration tool for highly structured data. Virgilio visualizes the results of a query to a database by generating VR scenes, that exploit appropriate metaphors in order to take advantage of common knowledge about real world objects, thus reducing the cognitive load in the process of information assimilation. The demonstration outlines an example scenario that applies the Virgilio prototype with an electronic commerce application.
\end{abstract}

\section{Keywords \\ Multimedia Database, User Interaction, Visual Metaphors, Information Visualisation, Virtual Reality}




\section{INTRODUCTION}

In many of nowadays web-based environments for electronic marketing and commerce, that present large multimedia product and service catalogues, it becomes more and more difficult to provide naive end users, such as private consumers or commercial business partners, with intuitive user interfaces to access the large multimedia collections describing the presented products and services. The same holds for marketing managers and other employees responsible for managing and maintaining the large and constantly changing set of multimedia information chunks and fragments contained in these collections.

As a consequence, many efforts are devoted to improve the quality of the interaction between users and databases. Virtual Reality (VR) techniques are a promising interaction paradigm particularly suited to novice and/or occasional users. The users are facilitated in the database navigation since the system proposes them an environment that reproduces a real situation and gives the possibility of interacting by manipulating objects that have a direct correspondence with known objects.

VR techniques combine the advantages of 3D visualizations with the power of metaphorical representations. Presenting the result of a database query through a VR scene allows users to explore data more easily since they interact with familiar objects. The structural and dynamic properties of the objects in the virtual world, i.e. the way objects can be composed and can act themselves, are predictable since they belong to the users' general background. No particular training should be required to interact with and explore the dataset, thus reducing the learning overhead of naive users when accessing information.

Virgilio is a VR based system that has been designed to be a general purpose exploration tool for highly structured data. It is capable of visualizing large sets of objects of considerable intra-object and inter-object complexity through effective VR techniques. Virgilio is based on several metaphors in order to take advantage of common knowledge about real world objects, thus reducing the cognitive load in the process of information assimilation. The overall system has been presented in (Massari et al., 1997), where lot of emphasis was posed on the generation of the 3D scenes, once the metaphor exploited in the visualization was chosen in a semiautomatic way, i.e. with the intervention of the system administrator. In this demonstration we present how visual metaphors for supporting user interaction with multimedia databases by means of virtual reality information visualisations can be automatically generated in Virgilio.

\section{THE APPLICATION SCENARIO AND THE APPROACH}

In order to allow users easy access to a database, the information stored in the database needs to be visualized in an information space. This visualization can 
either be carried out by the user in the user's mind, in which case it is essentially the user's conceptualization of the database; or the visualization could be accomplished by the system, in which case the visualization is generated on the display screen. The latter is what it is actually defined information visualization, i.e. "a process of transforming information into a visual form enabling the user to observe information" (Gershon et al., 1997). The essence of this process is to visually present information that is non inherently visual, such as text. Recent research has proved that successful visualization can reduce the time to get information, and to make sense out of it; it also enhance creative thinking.

Database objects, in general, are abstracted from real-life objects in the real world. Therefore, we can distinguish the logical information space and the physical information space (Chang and Costabile, 1997). In the logical space, the abstract database objects are represented. In the physical space, the abstract database objects are materialized and represented as physical objects that reflect real-life objects, such as diagrams, icons and sketches. For example, each object is materialized as an icon, and the physical information space consists of a collection of icons. These icons can be arranged spatially, so that the spatial locations approximately reflect the relations among database objects.

To create visualizations, the information in the logical space must be mapped into a physical space that will represent relationships contained in the information faithfully and efficiently. In this way, users will exploit their innate abilities to understand spatial relationships, also shifting most cognitive processing load to the perceptual system.

In the physical information space, the objects reflect real-world objects, but the world is still an abstract world. One further step is to present information in a VR information space. VR allows the users to be placed in a 3D environment they can directly manipulate. What the users see on the screen will be the same as what can be experienced in the real world. 3D features can be used to present the results in a VR setting. For example, if the database refers to the books of a library, we can represent a Virtual Library in which the physical locations of books are indicated by blinking icons in a 3D presentation of the book stacks of the library. What the user sees on the screen will be the same (after simplifications) as what can be experienced in the real world.

It is worth noting that we are talking about "nonimmersive" VR (Robertson $e t$ al., 1993), that is the user is placed in a 3D environment he/she can directly manipulate without wearing head-mounted stereo displays or special gloves, but acting only with mouse, keyboard, and monitor of a conventional workstation.

The real world, from which the database objects are abstracted, is the environment that the database objects must relate to. The real world is often abstracted in the information space. Only in the VR information space will the real world be represented in a direct way. Indeed, finding a good spatial representation of the information at hand is one of the most difficult tasks in visualization of 
abstract information. The key problem in information visualization is to invent visual metaphors for non physical data (Gershon et al., 1997).

In Virgilio, the mapping process described above is performed by the Metaphor Definition Tool (MDT). The MDT takes in input both the background knowledge on the virtual worlds and the Prolog query, and produces a metaphor graph that associates each node of the structure tree with some virtual object. The Virtual World Object Repository is the database where background knowledge on the virtual worlds is stored, while the Query Repository stores the structure trees and their corresponding Prolog queries. The metaphor graph is stored in the Metaphor Repository, so that it can be retrieved by the Scene Constructor Server that builds the sequence of scenes of the chosen virtual world that visualizes the query results. In the following, we will outline an example exploration of a virtual reality scene generated from the result of a query. It will be demonstrated how the corresponding visual representation can be explored interactively by users.

\section{VISITING AN EXAMPLE SESSION}

After an exemplar query has been executed and the result was retrieved from the database, a corresponding set of scene graphs is built by the Virgilio system. The first virtual reality object by the chosen metaphorical mapping mechanism in the first scene is a builiding. It represents the whole result of the query and is the starting point for the exploration.

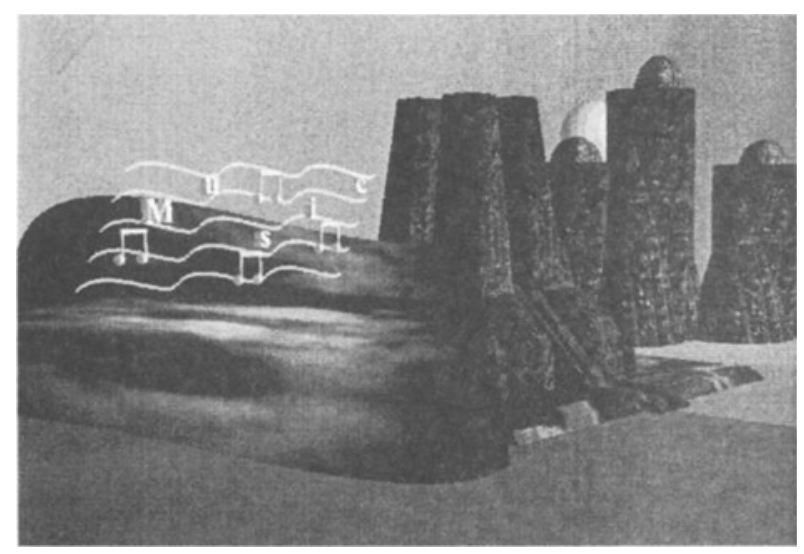

Figure 1. First scene visualising a "building" containing the query result. 
Inside the building the entrance hall aggregates different decorative objects and one container object, the elevator;

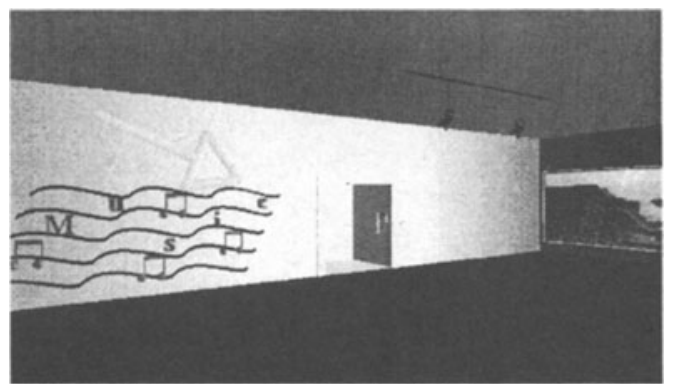

Figure 2. Second scene inside the "building"

The container object classifies different musical styles, providing an access to the next level of aggregation in the structure tree of the example query.

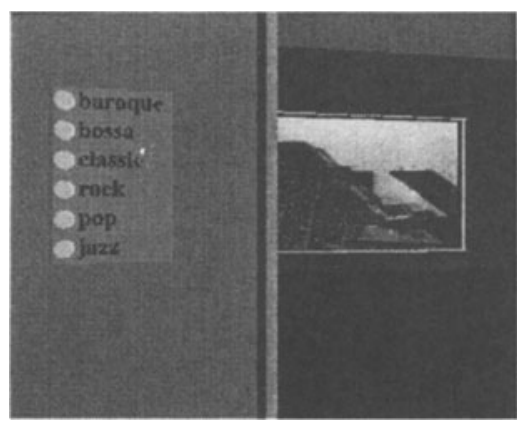

Figure 3. A container object classifying musical styles

After selecting a button and stepping out of the elevator, a poster reminds about the content of the chosen floor. The following corridor provides access to the rooms containing further information about different musicians.

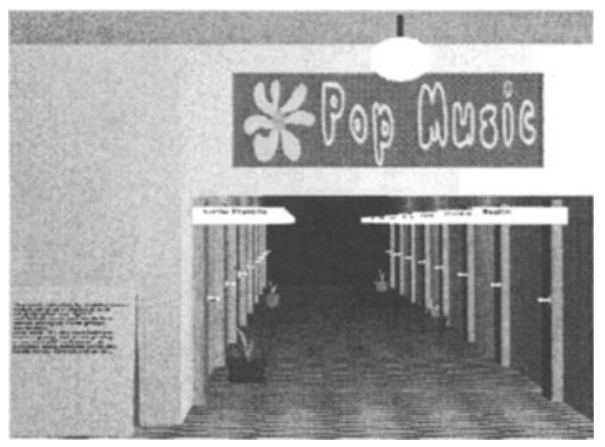

Figure 4. A "corridor" container object classifying musicians 
Entering a room users can see different objects representing the information related to the choosen musicians.

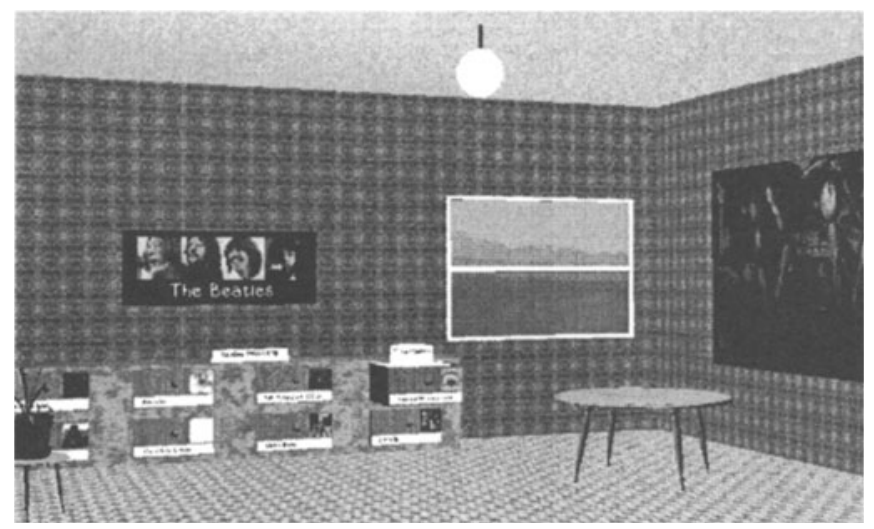

Figure 5. A "room" and other container objects visualizing and classifying information about musicians

\section{REFERENCES}

Chang, S.K. and Costabile, M.F. (1997) Visual Interface to Multimedia Databases, in The Handbook of Multimedia Information Management (eds. W.I. Grosky, R. Jain, and R. Mehrotra), Prentice Hall, Upper Saddle River, NJ, 167-187.

Gershon, N., Card, S. and Eich, S.G. (1997) Information Visualization, in Chi 97 Tutorial Notes, Atlanta, USA, 22-27 March 1997.

Massari, A., Saladini, L., Hemmje, M. and Sisinni, F. (1997) Virgilio: A NonImmersive VR System To Browse Multimedia Databases, Proceedings of the IEEE International Conference on Multimedia Computing and Systems, IEEE Computer Society Press, Los Alamitos, CA, 573-580.

Robertson, G.G., Card, S. K. and Mackinlay, J.D. (1993) Nonimmersive Virtual Reality, IEEE Computer, 26(2), 81-83. 


\section{BIOGRAPHY}

Maria F. Costabile received the Lurea degree in Mathematics at the Universita' della Calabria. Since 1989 she is associate professor at the Department of Computer Science of the University of Bari, Italy. From 1978 to 1988 she worked at the Dipartimento di Matematica, Universita' della Calabria. She has been visiting scientist in several foreign universities. Her current interests include theory of visual languages, visual interfaces, visual languages for querying databases, human-computer interaction, usability of interactive systems, user models. She has published several papers on the above topics, and edited four books. She served in committees of international conferences, and as program co-chair of AVI'96 and AVI'98. She is member of ACM, IEEE, AICA, IAPR. She is chairing the Italian Chapter of ACM SIGCHI.

Matthias Hemmje is a member of the OASYS information systems research division at GMD-IPSI in Darmstadt, Germany. He is working in various projects related to object-relational Multimedia Database Management Systems. Currently, he is responsible for R\&D of the ICE DataBlade Module (a database supported Information Catalogue Environment enabling navigation on multimedia documents), as well as a VRML-, an MPEG-, and an SGML- DataBlade Module. $\mathrm{He}$ has been conducting the design and development and evaluation of LyberWorld and VIRGILIO prototypes, both 3D graphical information visualization systems. Besides Multimedia Information Systems, his research interests include computer human interaction and information visualization for information systems.

Donato Malerba received the Laurea degree in Computer Science from the University of Bari, Italy, in July 1987. In 1991 he joined the University of Bari where he currently holds the rank of Assistant Professor in the Department of Informatics. In 1992, he has been a visiting scientist at the Department of Information and Computer Science, University of California at Irvine. His research interests are in machine learning, artificial intelligence and pattern recognition. Applications include document classification and understanding, knowledge discovery in databases, map interpretation and interfaces. He has published more than forty papers in international journals and refereed conference proceedings.

Aldo Paradiso received the Laurea degree in Computer Science from the University of Bari, Italy. He has been employed at the GMD-IPSI Institute in Darmstadt, Germany, where he is currently working on his $\mathrm{PhD}$ thesis. During his stay at GMD he has worked on the Virgilio Project, also creating a prototype of the system. His research interests include information visualization, particularly using Virtual Reality approaches. At the present he is working on Human Modeling using VR approaches. 